\title{
Equality in cleft and craniofacial care
}

\author{
Nicholas D. Sharratt ${ }^{1}$, Jean Calleja Agius ${ }^{2}$, Gareth Davies ${ }^{3}$, Felicity V. Mehendale ${ }^{4}$, Peter Hagell ${ }^{5}$, Martin \\ Persson $^{5}$ \\ ${ }^{1}$ Centre for Appearance Research, University of the West of England, Bristol BS16 1QY, UK. \\ ${ }^{2}$ Department of Anatomy, Faculty of Medicine and Surgery, University of Malta, Msida MSD2080, Malta. \\ ${ }^{3}$ European Cleft Organisation, Rijswijk ZH 2288 EL, The Netherlands. \\ ${ }^{4}$ Global Cleft Lip and Palate Research Programme, Global Health Research Centre, Usher Institute, University of Edinburgh, \\ Edinburgh EH8 9AG, UK. \\ ${ }^{5}$ Faculty of Health Sciences, Kristianstad University, Kristianstad SE-291 88, Sweden.
}

Correspondence to: Prof. Martin Persson, Faculty of Health Sciences, Kristianstad University, Kristianstad SE-291 88, Sweden. E-mail: martin.j.persson@hkr.se

How to cite this article: Sharratt ND, Calleja Agius J, Davies G, Mehendale FV, Hagell P, Persson M. Equality in cleft and craniofacial care. Plast Aesthet Res 2020;7:35. http://dx.doi.org/10.20517/2347-9264.2020.99

Received: 29 Apr 2019 First Decision: 12 Jun 2020 Revised: 19 Jun 2020 Accepted: 29 Jun 2020 Published: 12 Jul 2020

Academic Editor: Carroll Ann Trotman Copy Editor: Cai-Hong Wang Production Editor: Tian Zhang

\begin{abstract}
This review examines the issue of equality of care amongst those with cleft lip and/or palate in the European Union (EU) and beyond. Issues of equality both between and within national populations are considered, and it is argued that those from countries with smaller healthcare expenditure and who are from marginalised groups are at the greatest risk of, and affected most severely by, healthcare inequalities. The socioeconomic impact of inequality is also discussed. Having reviewed these topics, the goals and activities of the European Cleft and Craniofacial Initiative for Equality in Care Action, formed pursuant to an award from the EU's European Cooperation in Science and Technology, are introduced. Constituted of an open network of clinicians and researchers committed to exploring and reducing such inequalities, the ongoing Action is formed of multiple working groups examining these issues within the EU and has organised training schools, conferences and short-term scientific missions concerned with these issues. These activities are discussed along with the future directions of the Action, the impact it has had to date and the benefits of the European Cooperation in Science and Technology award.
\end{abstract}

Keywords: Cleft, craniofacial, equality, care, healthcare, Europe

\section{INTRODUCTION}

The European Union (EU) currently consists of 27 countries with a combined population of 446 million inhabitants ${ }^{[1]}$. There are approximately 637,000-743,000 individuals living with a cleft lip and/or palate (for convenience referred to hereafter as "cleft") in Europe and many others with other craniofacial conditions. 
Throughout their childhood and into their adult life, these individuals may benefit from the expertise of and care from a variety of specialists including surgeons (who may perform primary cleft surgery, revision surgery and further aesthetic surgeries) but also dentists, orthodontists, geneticists, nurses, paediatricians, psychologists, speech and language therapists, but their access to healthcare can vary greatly. For the general population in Europe, there is a significant variance in access to healthcare. When looking at the health expenditure per inhabitant in 2016, the countries with the lowest health expenditure were Romania $(€ 400)$, Bulgaria (€600) and Poland (€700). In contrast, the countries with the highest expenditures were Luxembourg (€5600), Sweden (€5100) and Denmark (€5000 $)^{[2]}$. The difference in resources can have an impact on the provision of care for cleft and craniofacial conditions and can therefore be important in determining health and social outcomes. In other words, the health and social outcomes of an individual with a cleft or craniofacial condition are dependent on the country in which they were born.

It is established that access to effective treatment for cleft and other craniofacial conditions varies widely throughout Europe, meaning that many children born with these conditions are never given the opportunity to realise their full potential. The concept of a comprehensive multi-specialist-team approach to care is not universal. Furthermore, infants with clefts are still institutionalised in some EU countries. A survey carried out for UNICEF in Bulgaria in 2011 showed that, in up to 40 per cent of cases where babies were born with a cleft, the parents were advised (in most cases by health professionals) to leave their baby in an orphanage. Via a fruitful collaboration, the European Cleft Organisation (ECO) and The Association of Children with Facial Anomalies and their Parents in Bulgaria (ALA) has helped reduce these numbers to 28 per cent through educating and training "front line" healthcare providers and families with children with facial anomalies. The work is ongoing ${ }^{[3]}$.

Clinicians and patients across Europe, especially in those regions that have limited healthcare expenditure, state that access to the necessary care pathways during the treatment span (from the moment of diagnosis to adulthood) is fragmented or in some cases non-existent in their countries. The importance of the early and adequate provision of information and treatment of clefts has been emphasised by the 2015 report of the European Committee for Standardisation (CEN)-Early Care Services for Babies Born with Cleft Lip and/or Palate. This involved input from 12 European countries and detailed the first agreed set of European guidelines in early cleft care ${ }^{[4]}$. The minimum standards of care and best practice models presented in this document led to a European Science Foundation funded conference held in Bucharest in October 2015, attended by nurses working in the field of cleft in 17 European countries. Key outcomes of the meeting were the urgent demand for a Europe-wide programme of implementation of the guidelines together with protocols on how to evaluate progress, especially in Eastern Europe. In these respects, the outcomes mirrored those of the earlier Eurocleft project, involving 30 countries and 201 centres, which recommended the adoption of a common set of policy statements governing clinical practice for European cleft teams and practice guidelines detailing minimum standards of care ${ }^{[5]}$. These recommendations, however, were never implemented on a consistent, Europe-wide basis.

Further evidence of the urgent need to improve cleft and craniofacial care in these countries was presented at a subsequent European Science Foundation funded meeting at CEN in Brussels in March 2016 attended by user groups, politicians and doctors from 13 European countries. It was emphasised that there is a need to adhere to evidence-based guidelines in order to develop national protocols. It was noted that there is still a lack of coordinated general measures in cleft care at a national level in many European countries including prenatal diagnosis, genetic advice, national registry development, equal access to treatment and transparent rules for treatment. There is a lack of coordinated multidisciplinary team protocols, deficiencies in information provision, both for professionals and parents, and deficiencies in long-term management. For example, it has been shown that the intervention of multidisciplinary team management in cases of women whose foetuses were diagnosed with cleft lip/palate on prenatal ultrasound screening was associated 
with these women being more likely to decide to continue their pregnancy than would have been expected if such intervention was not received ${ }^{[6]}$.

At the same time, the European Pillar of Social Rights states that "Everyone has the right to timely access to affordable, preventive and curative healthcare of good quality" p. $21^{[7]}$. There have been significant improvements in health in Europe due to advancing healthcare systems; however, access to healthcare remains uneven across countries and social groups, according to an individual's socioeconomic status, place of residence, ethnic group and gender ${ }^{[8]}$. Furthermore, it has been established that the lack of coverage and provision of certain types of care, such as cleft and craniofacial care, means that significant inequality remains in Europe. It is extremely important to acknowledge, however, that this inequality is not just between countries, but also within a country.

Inequalities are more present in socially marginalised groups; they are, for example (but not limited to), individuals who are unemployed, experience mental health problems, migrants or refugees and have disabilities. Belonging to a socially marginalised group also increases the risks of health inequalities, poverty and social exclusion. In 2017, 22.4 per cent of the European population (112.8 million people) lived in households at risk of poverty or social exclusion. In Bulgaria, Romania and Greece, more than a third of the population was at risk ${ }^{[9]}$. When it comes to children, figures for 2018 show they are the group at highest risk of poverty or social exclusion in Europe ${ }^{[10]}$.

A fundamental factor that explains inequalities in healthcare is that the lower the income of an individual or family is, the more self-reported unmet healthcare needs they have. A descriptive example is that in Greece those who are in the lowest income quintile report 34.3 per cent unmet healthcare needs while those in the highest quintile report only 0.4 per cent ${ }^{[8]}$. Currently, there exists evidence that large income differences result in poorer health and more negative social consequences ${ }^{[11]}$ and health inequities are increasing in many countries ${ }^{[12]}$. One of the factors that contributes to belonging to a group that is at risk of being in poverty and social exclusion is an individual's level of education ${ }^{[13]}$, since higher educational attainment often correlates with higher employment rates and higher earnings ${ }^{[14]}$. It also depends on which region an individual resides, since the 2018 unemployment rates in EU regions vary from $1.3 \%$ to $35.1 \%{ }^{[15]}$.

At the same time, research shows that individuals with cleft are at an elevated risk of poor educational outcomes in comparison to their peers ${ }^{[16-19]}$. This carries the potential to influence the long-term outcomes for individuals with cleft, since they are then hypothetically at a higher risk of poverty and/or social exclusion if they do not succeed in their education.

Experiencing inequality, poverty or social exclusion is also associated with mental health problems. Currently, in Europe, more than one in six people experience mental health problems ${ }^{[20]}$, and, when it comes to mental health problems for individuals with cleft, there are population-based studies from Denmark and Sweden that show a significantly increased risk for having psychiatric and neurodevelopmental disorders $^{[21,22]}$ as well as increased risk for being prescribed psychotropic medication ${ }^{[23,24]}$.

When individuals with a cleft or craniofacial conditions are seen by their healthcare providers, based on EU data for the general population, approximately 22 per cent are at risk for poverty or social exclusion ${ }^{[25]}$. However, the real figures are probably higher since the research introduced above shows that some individuals with a cleft are at significant risk of not doing well academically and at risk of mental health problems, which are associated with a higher risk of experiencing inequality, poverty and/or social exclusion. The impact is also influenced by the access to healthcare, as well as the economic situation in the country in which an individual with a cleft or craniofacial condition resides. 
The European Cleft and Craniofacial Initiative for Equality in Care (ECCE) is a pan-European multidisciplinary network with participants from hospitals and research institutions located in 27 European countries and beyond. ECCE is a 4-year project funded by the European Cooperation in Science and Technology (COST), promoting research and the development of innovation networks by providing support for arranging meetings, training schools and short-term scientific missions (STSMs) within the focus of the ECCE Action.

The ECCE Action is focusing on sharing scientific knowledge on treatment, research methods and organisational implementation in the different disciplinary groups in cleft and craniofacial care between the participants in the Action. The Action is made up of working groups to provide a structured environment within which mentoring and guidance will be offered to all the participants, with a particular focus on the early career investigators (ECIs) and researchers hailing from Inclusiveness Target Countries (ITC). This is aimed to facilitate the gaining of experience of leadership by participating in working group meetings and plenary sessions. In addition, as part of the COST framework, the Action organises conferences, short-term scientific missions and training schools targeting different aspects of equality in cleft and craniofacial care.

\section{THE EUROPEAN CLEFT AND CRANIOFACIAL INITIATIVE FOR EQUALITY IN CARE}

The activities that have been and are being undertaken as part of the ECCE Action are now introduced in order to consider how they contribute to the goals of the Action.

\section{Working groups}

To achieve a European perspective, the following areas are addressed by different working groups in the Action.

1. The primary level: the patient as the beneficiary

2. The level of the organisational context: the multidisciplinary team

3. The level of funding and policies: administration and resources

These areas are integrated with the imperative focus on the inequality of care in relation to healthcare resources, together with the impact for socially marginalised groups with cleft or craniofacial conditions. To address these objectives, the members of the Action have developed the following questionnaires during working group meetings in the first year of the Action.

1. Access to care: Asking questions about the ability to provide appropriate care for individuals with a cleft or craniofacial condition, based upon the respondents' experience where they work

2. Provision of care: Asking questions about the allocation of staff in the different specialities, available resources, number of new cases per year and funding modalities at their hospital

3. Patient organisations: Asking questions about perceptions of access to care amongst patient representative groups (e.g. whose members are primarily parents of children and young people with cleft or craniofacial conditions)

The analysis of the data is due to begin in the coming months.

Another activity undertaken in order to consider issues connected to equality of care was the use of Group Concept Mapping (GCM). GCM is a mixed qualitative and quantitative participant-driven method that aims to facilitate the understanding of complex phenomena, reveal their structures and discover new meaning ${ }^{[26,27]}$. The methodology comprises the generation of ideas (statements/items) through focus group brainstorming guided by a study-specific prompt that explicitly targets the focus or objective of the study. The ideas generated by the focus groups are then reviewed and edited (for clarity, redundancy, etc.) into a list of statements. This is followed by conceptual sorting and importance rating of the statements (alternative and additional ratings may be used). That is, participants individually sort the statements 
according to how they think they relate to one another, and then rate each statement's importance (with respect to the focus prompt) relative to all other statements. Sorted data are then analysed quantitatively (using multidimensional scaling) to map out relationships among individual statements, and cluster analysis is used to identify clusters of statements representing common aspects of the studied area. Finally, the map and its clusters are interpreted qualitatively together with rating data as a means to aid their use in, e.g., evaluation, planning and development. GCM has been used in a large variety of settings and purposes, including educational ones. For example, we have found GCM to be a valuable and very appropriate approach for evaluating and planning educational activities in higher education ${ }^{[28]}$ and for assessing the value of educational interventions for healthcare professionals ${ }^{[29]}$.

For ECCE, the first phase of the GCM involved the generation of statements and short sentences in relation to this question: "An important thing to help improve equality of care is..." These statements and short sentences were generated by healthcare professionals and members of the ECCE Action attending the ECCE meeting in Kristianstad, Sweden, on 14 December 2019. The second phase, which involves the conceptual sorting and importance rating of the statements generated in the first phase, is ongoing.

In the final year of the Action, a comprehensive plan for a minimum standard of multidisciplinary cleft and craniofacial care will be developed based on the outcomes from the areas mentioned above as well as the COST Action members' feedback. The creation of this standard will take into account the following aspects of health system goals.

1. Health: Improving the health condition for all individuals affected by cleft and craniofacial conditions

2. Responsiveness: In this context, all affected individuals and their families should have the same right to comprehensive treatment in Europe; hence, it is paramount to include differences concerning economic, social, demographic and other factors

3. Fair financing: All affected individuals should have access to comprehensive care and should not become impoverished or pay a disproportionate share of their income in obtaining needed healthcare or forfeit it because they cannot afford it

The envisioned benefits from scientific and socioeconomic perspectives are:

Scientific perspective

1. The ECCE Action will establish a sustainable network of European scientists and clinicians dedicated to research on how to provide and deliver the best care for families affected by cleft and craniofacial conditions.

2. The ECCE Action will agree on study designs in the different specialities for cleft and craniofacial research. This will enable studies to be undertaken with comparable models and comparable assessment tools.

3. The ECCE Action will facilitate enhanced research in this area across Europe, especially in those countries that have low healthcare expenditure. This has the potential to improve the provision of care for the families affected by cleft and craniofacial conditions.

4. The ECCE Action will increase the knowledge and skills of researchers and clinicians through their participation in the Action's activities.

5. The ECCE Action will enable a direct comparison of cleft and craniofacial care in Europe via the participants involved in the Action.

\section{Socioeconomic perspective}

1. The ECCE Action will facilitate the exchange and dissemination of knowledge on the effects of having an adequate provision of care for families with cleft and craniofacial conditions. This will help clinicians in each of the multidisciplinary team mentioned specialities to implement appropriate care, informed by evidence-based practice. 
2. The ECCE Action complements the European strategy of developing sustainable actions ${ }^{[30]}$.

3. The ECCE Action will develop and share knowledge on what works for whom and will facilitate improvements in cleft and craniofacial care, increase the chances of equal access to care.

4. The ECCE Action will actively facilitate interaction between clinicians, scientists and policymakers in seeking ways of ensuring that all affected families have the right to receive the minimum standard of care in all of the participating COST countries.

5. The ECCE Action could bring about substantial cost savings in relation to joint research activities across Europe.

6. The ECCE Action will increase the capacity for research by generating awareness amongst policymakers of the need for research into the cleft and craniofacial care and healthcare integration.

7. Since congenital anomalies do not discriminate against anyone, they also affect socially disadvantaged target groups, such as immigrants, the unemployed and the Roma population; thus, the ECCE Action addresses key areas of European focus such as disability ${ }^{[31]}$, children's rights ${ }^{[32]}$ and social exclusion ${ }^{[33]}$.

\section{Training schools}

An integral part of the ECCE Action is the organisation of training schools. Four training schools are planned, and they relate to the objectives of the different working groups. To date, one training school has been held, with the second one postponed in view of the COVID-19 pandemic.

The first training school was held in March 2019 in collaboration with the University of Malta, Valletta. This training school focused on the clinical and research-oriented approaches of integrated healthcare in relation to the decision-making process related to the diagnosis and treatment of patients. Since the patient is the primary beneficiary, this school trained the participants to facilitate patient empowerment programmes to make the patient feel he/she is an equal partner in decisions made around his/her care and have the confidence to pursue his/her own life goals unhindered by the stigma of his/her condition ${ }^{[34]}$.

The second training school is planned for October 2020 and will be held in Estonia. The focus will be on equality of care within the context of multidisciplinary teamwork, organisational structures and the implementation of change and will include training in the Health Innovation, Implementation and Impact (HI3) concept ${ }^{[35]}$. Further details of both these training schools are included within Supplementary Materials 1 . The final two training schools will be held during the final year of the Action.

\section{Conferences}

The ECCE Action conferences are free to attend and are vital to the Action's activities. Three major conference events are scheduled during the life of the Action, which bring together a rich and diverse network of researchers and stakeholders: user groups (beneficiaries); clinicians; health service providers (hospitals and health system coordinators); experts in public health medicine; representatives from health ministries; health economists; NGO's; and political lobbyists and activists. These events enable knowledge sharing at all levels, which is key to the Action's outputs and integral to promoting and facilitating dissemination. They are attended by local healthcare professionals, trainees, researchers and students in order to increase general awareness of and engagement with the issues discussed. They help cement research relationships between the COST participating countries leading to new connections and relationships, which enhance future research funding opportunities, sustaining the long-term effectiveness of treatment for all patients with orofacial clefts and other craniofacial conditions.

To date (June 2020), two conferences have been held. The first action conference was held in Niš in Serbia in 2018 and the second in Kristianstad, Sweden in late 2019. Further details are included in Supplementary Materials 2. The final Action summit will take place in Bucharest, Romania in September 2021, with a focus on the Action's outputs and recommendations and, importantly, promote the sustainability of the network(s) beyond the life of the Action. 


\section{Short-term scientific missions}

One of the benefits of COST Actions is the availability of STSMs, described by COST as a networking tool that encompasses a cross-border visit or exchange from one member of an Action to another. Typically, the subject matter of an STSM is connected to the Action or to some facet of the Action and, in turn, this facilitates future collaboration and the mutual sharing of techniques or ideas that might not otherwise be available or apparent.

The ECCE Action has the resources to offer approximately six STSMs per year of the Action, each of at least five working days duration. In tandem with the focus of this Action upon equality, the intention was that at least 75 per cent of these STSMs would be undertaken by ECIs or members of the Action based in ITCs and that there would be an equal gender split amongst the successful applicants.

To undertake an STSM, applicants (the "visitor") develop a proposal in collaboration with their proposed host and submit this to the Action's core group, who have been delegated the responsibility of considering and approving applications. To date (April, 2020), despite having to postpone one STSM as a result of the COVID-19 pandemic, the Action has supported 12 STSMs. Of these, 10 have been undertaken by members who satisfy the criteria of being an ECI and/or who are based in an ITC. There has also been an equal gender split, reflecting the balanced composition of the membership of the ECCE Action.

As a result of the applied nature and focus of the ECCE Action, a high proportion of its membership is clinically active. This has meant that the Action's STSMs have been well placed to benefit both research into cleft and craniofacial conditions as well as clinical and practical implications and applications. Example STSMs are described within Supplementary Materials 3.

It is also worth noting that the core group of the ECCE Action believed that any STSM that may have the potential to improve cleft care or our understanding of the condition was potentially within the remit of the Action. This is especially pertinent as cleft care is complex, can involve a large number of inter-connected disciplines, whether or not professionals are formally organised into multidisciplinary cleft teams, and can therefore be difficult for an individual to navigate, with their socioeconomic status and health literacy likely to be important factors in determining their ability to do so.

As the examples provided in Supplementary Materials 3 illustrate, the STSMs supported by the ECCE Action have covered a diverse array of topics, all undertaken with the desire to improve our understanding of and care for cleft and craniofacial conditions; to allow applicants and hosts to learn from one another and take that learning back to their local environment, share it and apply it; to foster collaborations and relationships that will endure beyond the life of the specific Action; and, ultimately, to improve and equalise access to care for cleft and craniofacial conditions. The members of the Action remain excited to follow the ongoing outcomes from the STSMs that have been performed to date, to discover what proposals will be submitted in the future and to develop the relationships built as part of the Action, at least in part attributable to the availability of STSMs.

\section{CONCLUSION}

It is well established that disparities and inequalities exist within healthcare in the EU and subsist within cleft and craniofacial care. These extend to health expenditure, access to effective and multidisciplinary healthcare following a clear treatment pathway or protocol, the provision of long-term case management and the operation of national data registries. Such disparities exist between countries and within subsets of a national population. In turn, this may prejudice patients' social and economic opportunities and, in cleft care, compound the impact of their condition on educational attainment and socioeconomic status. 
Whilst the activities of the ECCE Action are ongoing, it has already enabled over 135 researchers and clinicians from 30 countries to attend one or more of the events facilitated by the Action, including training schools, short-term scientific missions and conferences, all related to the topic of equality in care. It is believed by the authors that this exposure to the experiences and healthcare cultures of such a diverse group of researchers and clinicians can only serve to increase awareness of this topic amongst those researchers, clinicians and trainees involved in the Action. In addition to facilitating individual awareness and, potentially, enabling those working clinically to carry this awareness into their practice, those involved in the Action are committed to sharing their learning with colleagues and, gradually, instigating organisational change at a local level in order to combat healthcare inequalities where possible.

The activities of the working groups, including the surveys and questionnaires and GCM exercises being undertaken, the STSMs and the long-term collaborations forged in performing this activity together as well as during conferences and training schools, will also lead to contributions to the literature on this topic. This collaboration between participants in the ECCE Action has resulted in four ongoing research or pilot endeavours concerning the access to and provision of care in cleft and craniofacial conditions and has generated six further EU grants comprising a total of 16 different country partners and a combined grant sum of 1,372,000 Euros. In turn, it is hoped that the immediate outputs of the ECCE Action as well as those stemming from partnerships it has helped create will increase awareness and encourage reflection, action and application within the clinical, research and healthcare policy spheres. These outputs will also be important in establishing equality of access to cleft and craniofacial care as a critical issue going forward and maintaining a focus on the importance of equality of care.

These achievements should be understood against the background of it being a significant challenge to attract funding to enhance knowledge of relatively rare medical conditions such as cleft and other craniofacial anomalies. In this respect, COST provides networking opportunities for researchers and innovators to strengthen Europe's capacity to address scientific, technological and societal challenges. The principal areas of activity are promoting and spreading excellence, fostering interdisciplinary research for breakthrough science and empowering and retaining young researchers and innovators. COST implements its mission by funding bottom-up, excellence-driven, open and inclusive networks in all areas of science and technology. Whilst COST does not fund research time, it provides support for networking activities carried out within Actions and, as has materialised with the ECCE Action, increases the possibility of the topic under consideration being the subject of further applications for research funding.

The fact that COST puts significant emphasis on helping early career researchers and clinicians grow professionally is both unique and very important for topics such as equality of care. This is because there exists an opportunity to influence and shape future approaches to cleft and craniofacial care and research in Europe. For these groups, the training schools and short-term scientific missions have been, and will continue to be, powerful tools that can act as an introduction to cross-border collaboration and highlight future possibilities. Furthermore, COST also prioritises clinicians and researchers from less researchintensive countries. This has been hugely beneficial in bringing together researchers, especially in those parts of Europe that have hitherto not had the opportunity to exchange knowledge and ideas with counterparts in other countries due to limited resources. Indeed, funding is not only open to Action members but also a limited number of colleagues and researchers in countries represented in the Action. Grants to attend the conferences and training schools and take part in STSMs are usually sufficient to cover most, if not all, of the costs of travel, accommodation and subsistence, which ensure networking opportunities are genuinely open to everyone eligible. In this sense, the ECCE Action has been able to encourage and foster opportunities and equality within its own members and its own activities, a critical requirement given the focus of this Action upon equality. 
A vital component of the Action is to generate awareness about the inequalities of care that exist between and within countries in Europe and beyond. In addition to the activities detailed, members of the Action are participating in dissemination actives, including key conferences around the world and with the European Parliament. The ECCE Action network is, however, not closed but remains open. Interested clinicians and researchers, from the EU and beyond, who wish to learn more and/or collaborate on this project or on future projects are invited to contact the corresponding author and discuss these possibilities.

\section{DECLARATIONS}

\section{Authors' contributions}

Substantially contributed to drafting and reviewing the manuscript: Sharratt ND, Calleja Agius J, Davies G, Mehendale FV, Hagell P, Persson M

Substantially contributed to the conception of this project and preparation of the funding application: Davies G, Persson M

\section{Availability of data and materials}

Not applicable.

\section{Financial support and sponsorship}

This project is supported by funding awarded by the EU's European Cooperation in Science and Technology programme, Action CA16234. The funding awarded supports the costs and expenses associated with the activities of the Action and the management of the Action. The time commitment required of members of the Action is supported by each individual member's employer and/or the dedication of their personal time to the Action. The employment of the first author is supported by a donation made to the University of the West of England by the Vocational Training Charitable Trust Foundation.

\section{Conflicts of interest}

All authors declared that there are no conflicts of interest.

\section{Ethical approval and consent to participate}

Not applicable.

\section{Consent for publication}

Not applicable.

\section{Copyright}

(c) The Author(s) 2020.

\section{REFERENCES}

1. European Union. EU in figures: Living in the EU: 2020. Available from: https://europa.eu/european-union/about-eu/figures/living_en [Last accessed on 29 Apr 2020]

2. Eurostat. Healthcare expenditure in the EU: 2018. Available from: https://ec.europa.eu/eurostat/web/products-eurostat-news/-/DDN20181129-2 [Last accessed on 3 Jul 2020]

3. European Cleft Organisation - 10 years in Bulgaria: 2019. Available from: http://europeancleft.org/wp-content/uploads/2020/04/ECO-inBulgaria-FINAL_1.pdf [Last accessed on 19 Jun 2020]

4. The European Committee for Standardisation. CEN/TR 16824: 2015 Early care services for babies born with cleft lip and/or palate. Available from: https://standards.iteh.ai/catalog/tc/cen/d0bb9258-df47-411a-a9e1-cd383fcfb5b9/cen-tc-424 [Last accessed on 8 Jul 2020]

5. Shaw WC, Semb G, Nelson P, Brattström V, Mølsted K, et al. The Eurocleft project 1996-2000: overview. J Craniomaxillofac Surg 2001;29:131-40.

6. Han HH, Choi EJ, Kim JM, Shin JC, Rhie JW. The importance of multidisciplinary management during prenatal care for cleft lip and palate. Arch Plast Surg 2016;43:153-9.

7. European Parliament, Council of the European Union, and European Commission. European Pillar of Social Rights. Available from: 
https:/ec.europa.eu/commission/sites/beta-political/files/social-summit-european-pillar-social-rights-booklet_en.pdf [Last accessed on 3 Jul 2020]

8. Baeten R, Spasova S, Vanhercke B, Coster S. Inequalities in access to healthcare. A study of national policies, European Social Policy Network (ESPN). Brussels: European Commission; 2018.

9. Eurostat. Archive: People at risk of poverty or social exclusion. 2017. Available from: https://ec.europa.eu/eurostat/statistics-explained/ index.php/People_at_risk_of_poverty_or_social_exclusion\#Number_of_people_at_risk_of_poverty_or_social_exclusion [Last accessed on 6 Apr 2020]

10. Eurostat. Children at risk of poverty or social exclusion. Available from: https://ec.europa.eu/eurostat/statistics-explained/index. php?title=Children_at_risk_of_poverty_or_social_exclusion\#The_highest_risk_of_poverty_or_social_exclusion [Last accessed on 6 Apr 2020]

11. Pickett KE, Wilkinson RG. Income inequality and health: a causal review. Soc Sci Med 2015;128:316-26.

12. Marmot M. Review of social determinants and the health divide in the WHO European Region: final report. Copenhagen:WHO Regional Office for Europe; 2014.

13. Keeley B. Human Capital: How what you know shapes your life. Paris: OECD Publishing; 2007.

14. U.S. Bureau of Labor Statistics. Unemployment rates and earnings by educational attainment. Available from: https://www.bls.gov/emp/ chart-unemployment-earnings-education.htm [Last accessed on 8 Apr 2020]

15. Eurostat. Unemployment statistics at regional level. Available from: https://ec.europa.eu/eurostat/statistics-explained/index.php/ Unemployment_statistics_at_regional_level\#Regional_unemployment_rates_and_the_EU_average [Last accessed on 8 Apr 2020]

16. Watkins SE, Meyer RE, Aylsworth AS, Marcus JR, Allori AC, et al. Academic achievement among children with nonsyndromic orofacial clefts: a population-based study. Cleft Palate Craniofac J 2018;55:12-20.

17. Fitzsimons KJ, Copley LP, Setakis E, Charman SC, Deacon SA, et al. Early academic achievement in children with isolated clefts: a population-based study in England. Arch Dis Child 2018;103:356-62.

18. Wehby GL, Collet B, Barron S, Romitti PA, Ansley TN, et al. Academic achievement of children and adolescents with oral clefts. Pediatrics 2014;133:785-92.

19. Persson M, Becker M, Svensson H. Academic achievement in individuals with cleft: a population-based register study. Cleft Palate Craniofac J 2012;49:153-9.

20. OECD. "More than one in six people in EU countries have a mental health problem" in Health at a Glance: Europe 2018: State of Health in the EU Cycle. Paris: OECD Publishing; 2018.

21. Pedersen DA, Wehby GL, Murray JC, Christensen K. Psychiatric diagnoses in individuals with non-syndromic oral clefts: a danish population-based cohort study. PLoS One 2016;11:e0156261.

22. Tillman KK, Hakelius M, Hoijer J, Ramklint M, Ekselius L, et al. Increased risk for neurodevelopmental disorders in children with orofacial clefts. J Am Acad Child Adolesc Psychiatry 2018;57:876-83.

23. Nilsson S, Merlo J, Lyberg-Ahlander V, Psouni E. Psychotropic drug use in adolescents born with an orofacial cleft: a population-based study. BMJ Open 2015;5:e05306.

24. Pedersen DA, Hageman I, Wehby GL, Christensen K. Use of psychotropic medications and visits to psychiatrists and psychologists among individuals with nonsyndromic oral clefts: a population-based cohort study. Birth Defects Res 2017;109:824-35.

25. Eurostat. Archive: People at risk of poverty or social exclusion. 2017. Available from: https://ec.europa.eu/eurostat/statistics-explained/ index.php/People_at_risk_of_poverty_or_social_exclusion\#Number_of_people_at_risk_of_poverty_or_social_exclusion [Last accessed on 06 April 2020]

26. Kane M, Trochim WMK. Concept mapping for planning and evaluation. Thousand Oaks: Sage Publications, Inc; 2007.

27. Trochim WMK. An introduction to concept mapping for planning and evaluation. Eval Program Plann 1989;12:1-16.

28. Hagell P, Edfors E, Hedin G, Westergren A, Hammarlund CS. Group concept mapping for evaluation and development in nursing education. Nurse Educ Pract 2016;20:147-53.

29. Westergren A, Edfors E, Norberg E, Stubbendorff A, Hedin G, et al. Computer-based training in eating and nutrition facilitates personcentered hospital care: a group concept mapping study. Comput Inform Nurs 2018;36:199-207.

30. European Commission Communication. EUROPE 2020 A strategy for smart, sustainable and inclusive growth. Brussels European Commission 2010. Available from: https://eur-lex.europa.eu/legal-content/en/ALL/?uri=CELEX\%3A52010DC2020 [Last accessed on 8 Jul 2020]

31. European Commission Communication. European Disability Strategy 2010-2020: A Renewed Commitment to a Barrier-Free Europe. Brussels: European Commission; 2010.

32. European Commission Communication. Early Childhood Education and Care: Providing all our children with the best start for the world of tomorrow. Brussels European Commission 2011. Available from: https://eur-lex.europa.eu/legal-content/EN/ ALL/?uri=CELEX\%3A52011DC0066 [Last accessed on 8 Jul 2020]

33. European Commission Communication. The European Platform against Poverty and Social Exclusion: A European framework for social and territorial cohesion. Brussels European Commission 2010. Available from: https://eur-lex.europa.eu/LexUriServ/LexUriServ.do?uri= COM\%3A2010\%3A0758\%3AFIN\%3AEN\%3APDF [Last accessed on 8 Jul 2020]

34. ECCE. Training School Malta. The European Cleft and Craniofacial Initiative for Equality in Care 2019. Available from: https://ecce.nu/ Malta-2019 [Last accessed on 8 Jul 2020]

35. HI3. Health Innovation, Implementation and Impact - A functional training program on how to implement sustainable change in the healthcare system on a clinical level. Available from: http://health-innovation.nu/ [Last accessed 15 Jun 2020] 